\title{
Phantom testing of two clinical spatially-resolved NIRS instruments
}

\author{
A.J. Macnab* and R.E. Gagnon \\ Department of Pediatrics, University of British Columbia, Vancouver, Canada
}

\begin{abstract}
We used a living biological phantom to evaluate whether previously observed clinical differences in data collection between two similar near infrared spectrophotometers were due to technical design differences. A Somanetics INVOS-5100 was compared to a Hamamatsu NIRO-300. Both express the ratio of oxygenated hemoglobin to total hemoglobin concentration as a percentage, and both are intended for monitoring cerebral oxygenation status at the bedside. The living phantom simulated different bone densities and contained a low haematocrit solution of blood, intra-lipid, and saline, to which was added grower's yeast to alter oxygenation status. Results: In all trials, oxygen saturation decreased from $100 \%$ to $0 \%$ as the yeast consumed the available oxygen. In all trials the spectrometers were significantly correlated (range: $r^{2}=0.815$ to $0.995, p<0.05$ for $r^{2}>0.232$ ). The NIRO-300 indicated oxygenation change 1 minute after addition of yeast, whereas the INVOS-5100 showed change after 5 minutes. Simulated bone thickness had almost no effect upon the NIRO-300, but did affect the INVOS5100. Conclusion: In this study, the spectrophotometers had similar results consistent with the technical design differences: the INVOS-5100 does not report hemoglobin saturation change above $95 \%$ or below $15 \%$ while the NIRO-300 reports the full range from $100 \%$ to $0 \%$.
\end{abstract}

\section{Introduction}

When similar medical devices are introduced in the clinical setting there is interest in comparing the devices to evaluate whether the devices can be used interchangeably. Proprietary design features and technical components ensure that, at a basic level, the devices are different, even though intended for the same clinical application(s). The comparison of similar clinical near infrared spectrophotometers (NIRS) is difficult because the light detector arrays have too much surface area to permit being positioned at the same time on the same tissue region. Further, if the NIRS detectors are placed exactly, but at alternate times, the underlying nanosecond biological reactions will perpetually alter the region's energy and respiratory dynamics, thereby making comparisons unreliable. In humans, bilateral symmetry is too inexact to ensure either precise lateral positioning or identical underlying tissue volumes and composition for exact dual sampling. The acute ability of the NIRS systems to detect photon flux, and biological variability, together imply that in vivo comparisons of clinical NIRS devices will be inconclusive.

In vivo comparisons of similar medical devices are difficult because of intra and inter subject physiologic variability. Apparent differences can as readily be attributed to the devices' independent technical characteristics as they can to the subjects' progressive pathology, general level of health, and/or momentary biological status. In vitro comparisons can isolate the pathology, health, and/or status confounders

\footnotetext{
${ }^{*}$ Corresponding author: Prof. Andrew Macnab, Director, Critical Care Research Office, Room L317, Mail Box 80, Children's \& Women's Health Centre, 4480 Oak St., Vancouver, BC, Canada V6H 3V4. Tel.: +1 (604) 875 3524; Fax: +1 (604) 875 2890; E-mail: amacnab@cw.bc.ca.
} 
but are flawed because many critical aspects of in vivo systems are absent from in vitro systems. Thus both types of comparisons are needed to fully evaluate similar devices.

Previously we reported the in vivo comparison of two clinical near infrared spectrophotometers, the Hamamatsu NIRO-300 (Hamamatsu KK, Hamamatsu City, Japan), and the INVOS-5100 (Somanetics Corp, Troy, MI, USA) [2]. Both devices use the principles of photon diffusion and spatially-resolved correction of path length to derive the absolute ratio between oxygenated hemoglobin and total hemoglobin concentrations expressed as a percentage. The NIRO-300 reports Tissue Oxygenation Index (TOI), and the INVOS-5100 reports Regional Oxygen Saturation Index (rSO2). The instruments use multiple, sequentially pulsed, 750-1000 nm laser diodes for transcutaneous illumination of tissue and detect refracted photons by dual and triple photodiode arrays. We found that although there was close agreement between the devices in response to major physiological change, the absolute values differed between devices throughout the range of measurements. However, we were unable to obtain simultaneous measurements of the same tissue regions because of the large size of the devices' emitter/detector arrays.

To determine whether the difference in absolute values was due to optical interference by the surrounding skull we used the same NIRS devices to conduct the current follow-up in vitro study. This study specifically used a phantom of suitable size to allow simultaneous measurements of a homogenous media. A difference in outcome between devices in this study could explain, in part, why differences occurred in the previous in vivo study.

\section{Method}

A rectangular phantom chamber, shown in Fig. 1, was constructed of epoxy resin with $10 \mathrm{~mm}$ and $5 \mathrm{~mm}$ thick opposing vertical walls to simulate different bone thickness. The wall material had a scattering coefficient of $1.0 \mathrm{~mm}^{-1}$ and absorption coefficient of $0.01 \mathrm{~mm}^{-1}$. The long axis vertical walls were

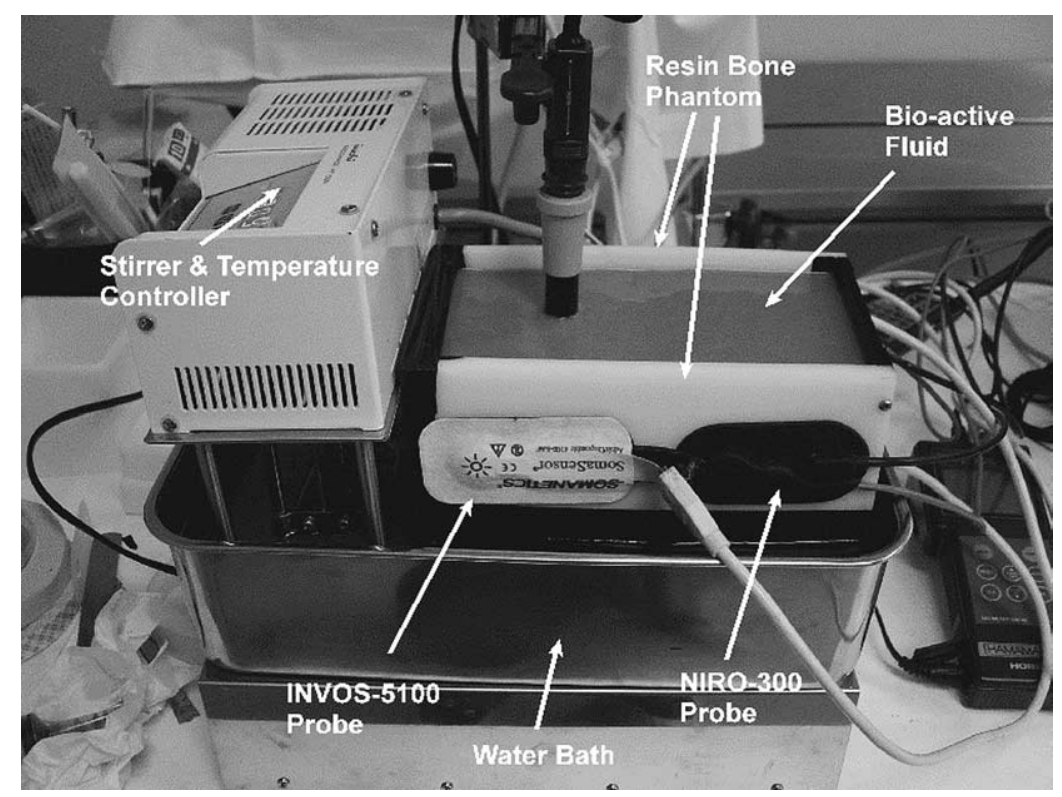

Fig. 1. Living phantom apparatus. Four NIRS probes are placed with two on each opposing bone phantom surface. The probes are connected to dual channel monitors that are not shown. 
$200 \mathrm{~mm}$ by $100 \mathrm{~mm}$, sufficient to accommodate two emitter/detector light shield arrays. Another two emitter/detector light shields, one from each spectrometer, were affixed to the opposite long axis vertical wall. The photon detectors on each wall were placed abutting each other while the near infrared emitters were oriented with as great a separation as possible. The short axis of the chamber was approximately $150 \mathrm{~mm}$, sufficient to ensure there was no crosstalk between the emitters.

The chamber was filled with $2200 \mathrm{ml}$ of fresh swine blood diluted to $1 \%$ haematocrit by $1 \%$ intralipid and normal saline. The chamber had an oscillating stirrer and was placed in a hot water bath to maintain the biological solution at $37^{\circ} \mathrm{C}$. The solution's oxygenation status was altered by adding $3 \mathrm{~g}$ of yeast, which actively consumed oxygen during cell respiration and reproduction. Change in oxygenation status was monitored intermittently by a hand-held dissolved oxygen meter with its probe immersed in the solution.

The ratio of oxygenated hemoglobin to total hemoglobin was monitored continuously at 5 seconds intervals by a NIRO-300 clinical spectrophotometer, and continuously at random intervals, between 7 and 17 seconds, by an INVOS-5100 clinical spectrophotometer.

Data were collected for 18 minutes in each of 3 trials. Linear correlation regression analysis was used to evaluate whether the measurements by either NIRS device were affected by the bone phantom.

\section{Results}

In each of three trials, oxygen saturation decreased from $100 \%$ to $0 \%$ saturation as the growing yeast consumed the oxygen. A typical data collection is shown in Fig. 2. The results of the linear correlation regression analysis are summarized in Table 1.

In all trials, there was a greater range of change observed via the $5 \mathrm{~mm}$ thick wall, then via the $10 \mathrm{~mm}$ thick wall regardless of NIRS device. Since the same moment of cessation of change was observed regardless of wall thickness or NIRS device, there was a greater rate of change observed via the $5 \mathrm{~mm}$ thick wall than via the $10 \mathrm{~mm}$ thick wall. The NIRO-300 a change in oxygen consumption beginning at the same time through both wall thicknesses, whereas the INVOS-5100 recorded the start of change through the $5 \mathrm{~mm}$ thick wall 120 seconds prior to that observed through the $10 \mathrm{~mm}$ thick wall.

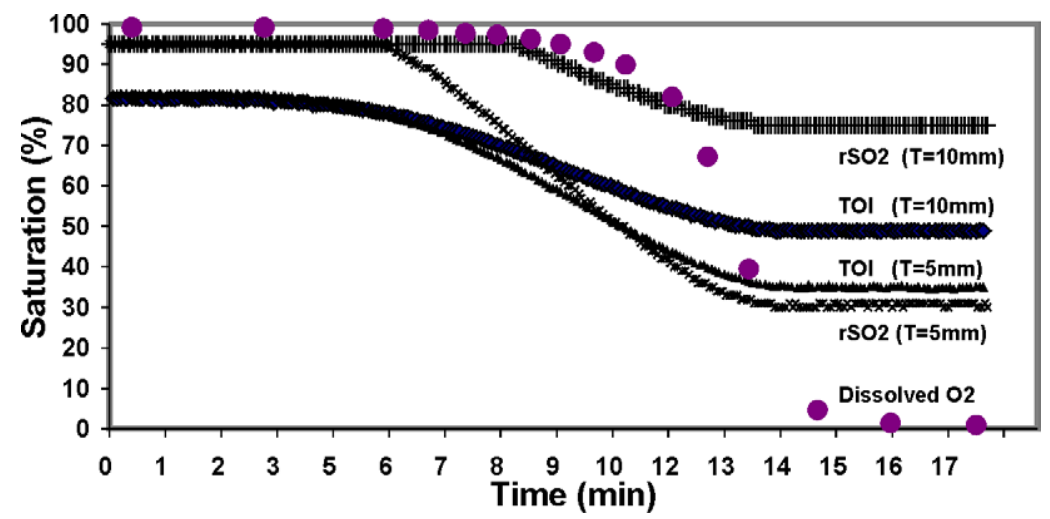

Fig. 2. Typical data collection where " $T=10 \mathrm{~mm}$ " and " $T=5 \mathrm{~mm}$ " represent the thickness of the epoxy resin bone phantom, "rSO2" is the INVOS 5100 saturation index, "TOI" is the NIRO-300 saturation index, and "Dissolved O2" is the dissolved oxygen content measured by an independent meter. 
Table 1

Linear correlation regression analysis values $\left(r^{2}\right)$ for relationships

\begin{tabular}{|c|c|c|c|}
\hline Analysis & Trial 1 & Trial 2 & Trial 3 \\
\hline TOI $(T=10 \mathrm{~mm})$ vs dissolved oxygen & 0.7381 & 0.6262 & 0.7517 \\
\hline TOI $(T=5 \mathrm{~mm})$ vs dissolved oxygen & (not collected) & 0.6427 & 0.7871 \\
\hline $\mathrm{rSO} 2(T=10 \mathrm{~mm})$ vs dissolved oxygen & 0.8925 & 0.7500 & 0.9006 \\
\hline $\mathrm{rSO} 2(T=5 \mathrm{~mm})$ vs dissolved oxygen & 0.8223 & 0.6282 & 0.8271 \\
\hline TOI $(T=10 \mathrm{~mm})$ vs TOI $(T=5 \mathrm{~mm})$ & (not collected) & 0.9994 & 0.9985 \\
\hline $\operatorname{rSO} 2(T=10 \mathrm{~mm})$ vs $\mathrm{rSO} 2(T=5 \mathrm{~mm})$ & 0.9318 & 0.9270 & 0.9295 \\
\hline TOI $(T=10 \mathrm{~mm})$ vs rSO2 $(T=10 \mathrm{~mm})$ & 0.8159 & 0.9191 & 0.8398 \\
\hline TOI $(T=5 \mathrm{~mm})$ vs $\mathrm{rSO} 2(T=5 \mathrm{~mm})$ & (not collected) & 0.9951 & 0.9755 \\
\hline
\end{tabular}

" $T=10 \mathrm{~mm}$ " and " $T=5 \mathrm{~mm}$ " represent the thickness of the epoxy resin bone phantom, "rSO2" is the INVOS 5100 saturation index, "TOI" is the NIRO-300 saturation index, and "Dissolved O2" is the dissolved oxygen content measured by an independent meter. The level of significance for all values is $p<0.05$ for $r^{2}>0.2324$.

In each trial, there were high correlations between the patterns of change obtained by both spectrophotometers (range: $r^{2}=0.815$ to $0.995, p<0.05$ for $r^{2}>0.2324$ ), and between both spectrophotometers and the dissolved oxygen meter (range: $r^{2}=0626$ to $0.901, p<0.05$ for $r^{2}>0.2324$ ). With either spectrophotometer, there were very high correlations between the simultaneous dual channel data collections of the $5 \mathrm{~mm}$ and $10 \mathrm{~mm}$ thick chamber walls (range: $r^{2}=0.927$ to $0.999, p<0.05$ for $\left.r^{2}>0.2324\right)$.

The NIRO-300 spectrophotometer indicated that the phantom solution's change in oxygenation status began at 60 seconds following the addition of yeast, whereas the dissolved oxygen meter showed change after 150 seconds, and the INVOS-5100 showed change after 300 seconds. Both spectrophotometers indicated cessation of change in oxygenation status 2 minutes before the dissolved oxygen meter measured $0 \%$ saturation.

\section{Discussion}

Our study applied in vitro techniques to provide insight into our previous in vivo study finding that although the oxygenation patterns of change reported by two different clinical NIRS devices were highly correlated, their momentary values were dissimilar [2]. The in vivo relationship between devices was found to be:

$$
\mathrm{TOI}=0.45(\mathrm{rSO} 2)+38.53
$$

Our current in vitro results also indicate that the data from the two devices are highly correlated, and therefore can be used to determine whether the single variable, simulated bone thickness, is the cause of difference in momentary values between the devices. Other investigations have compared these oxygenation indices via haemoglobin saturation change in order to validate the signals but did not express a correlation between devices, and thus could not yield insight into the relationship expressed above $[1,3,4,7]$.

Other investigators used Monte Carlo statistical modelling to predict that skull thickness affected the sensitivity of NIRS signals [5]. We found that both NIRS devices were affected by bone thickness in that the rate of change, and therefore the range of values, observed through the thinner wall was greater than that of the thicker wall. However, since the two fold difference in simulated bone thicknesses had almost no effect upon the pattern of change for the NIRO-300 $\left(r^{2}=0.999, p<0.05\right.$ for $\left.r^{2}>0.2324\right)$, 
and only a slight effect upon that of the INVOS-5100 $\left(r^{2}=0.927, p<0.05\right.$ for $\left.r^{2}>0.2324\right)$, and there was strong correlation between both devices when paired by wall thickness, bone density is an unlikely confounder in explaining our previous in vivo results where the variation in skull thickness between measurement sites was small.

The 240 second disparity between NIRS devices in reporting the start of change in oxygenation is likely due to the NIRO-300's ability to report the full range from $100 \%$ to $0 \%$ while the INVOS-5100 does not report the range above $95 \%$ or below $15 \%$, as the NIRO-300 did report the start of change earlier than the INOVS-5100. Also, our finding that both devices reported the cessation of oxygenation change at the same moment, and were highly correlated throughout the period of change, suggests that the disparity at the start of change is not persistent throughout the range of measurements.

Our outcomes indicate that the difference in observed values between these two spectrometers seen in our previous in vivo study of 24 healthy subjects, each of a different skull thickness, cannot be attributed to different sensitivity to skull attenuation [2]. Since the underlying physiology of those subjects was determined by genetic selection, age, and size, and affected by, controlled respiratory, circulatory, and metabolic interventions, the results of that study combined with those of the current in vitro study imply that the earlier finding that the two devices do not yield identical values is likely due to technical differences in the proprietary algorithms used to generate the values [2]. Other investigators comparing these same devices have reached a similar conclusion [6].

Assessing whether different makes of clinical NIRS devices can be used interchangeably is important for clinical safety, research design, regulatory approvals and cost efficiency. Our results indicate that the NIRO-300 and INVOS-5100 can be used interchangeably. This in vitro finding is consistent with that of our previous in vivo study.

\section{References}

[1] H. Cho, E.M. Nemoto, M. Sanders, K. Fernandez and H. Yonas, Comparison of two commercially available near-infrared spectroscopy instruments for cerebral oximetry, J. Neurosurg. 93(2) (2000), 351-354.

[2] R.E. Gagnon, A.J. Macnab, F.A. Gagnon, D. Blackstock and J. LeBlanc, Comparison of two spatially resolved NIRS oxygenation indices, J. Clin. Mon. Comp., Manuscript \#JOCM 2052-02 in press.

[3] C.D. Gomersall, P.I. Leung, T. Gin, G.M. Yoynt, R.J. Young, W.S. Poon and T.E. Oh, A comparison of the Hamamatsu NIRO 500 and the INVOS 3100 near-infrared spectrophotometers, Anaesth. Intensive Care 26(5) (1998), 548-557.

[4] G. Grubhofer, W. Tonninger, P. Keznickl, P. Skyllouriotis, M. Ehrlich, M. Hiesmayr and A. Lassnigg, A comparison of the monitors INVOS 3100 and NIRO 500 in detecting changes in cerebral oxygenation, Acta Anaesthesiol. Scand. 43(4) (1999), 470-475.

[5] E. Okada and D.T. Delpy, Near-infrared light propagation in an adult head model. II. Effect of superficial tissue thickness on the sensitivity of the near-infrared spectroscopy signal, Appl. Opt. 42(16) (2003), 2915-2922.

[6] M. Thavasothy, M. Broadhead, C. Elwell, M. Peters and M. Smith, A comparison of cerebral oxygenation as measured by the NIRO 300 and the INVOS 5100 near infrared spectrophotometers, Anaesthesia 57(10) (2002), 999-1006.

[7] K. Yoshitani, M. Kawaguchi, K. Tatsumi, K. Kitaguchi and H. Furuya, A comparison of the INVOS 4100 and the NIRO 300 near-infrared spectrophoptometers, Anesth. Analg. 94(3) (2002), 586-590. 


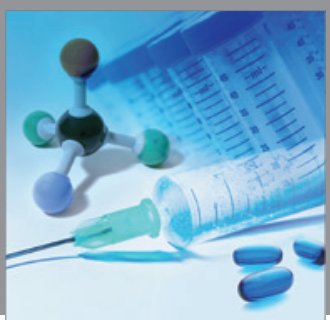

International Journal of

Medicinal Chemistry

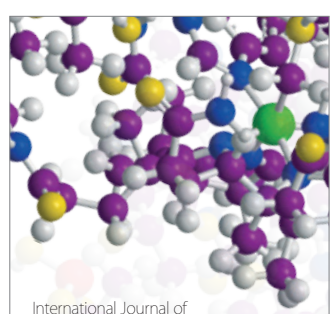

Carbohydrate Chemistry

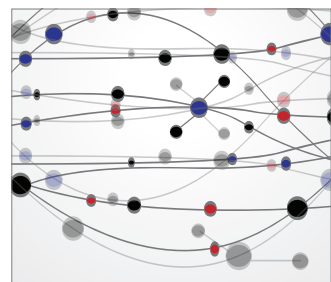

The Scientific World Journal
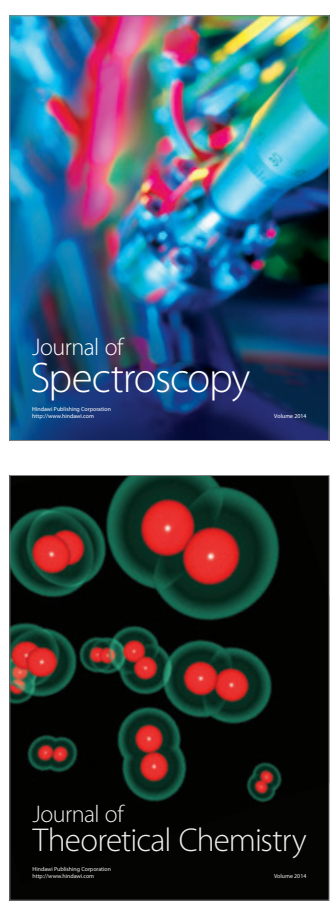
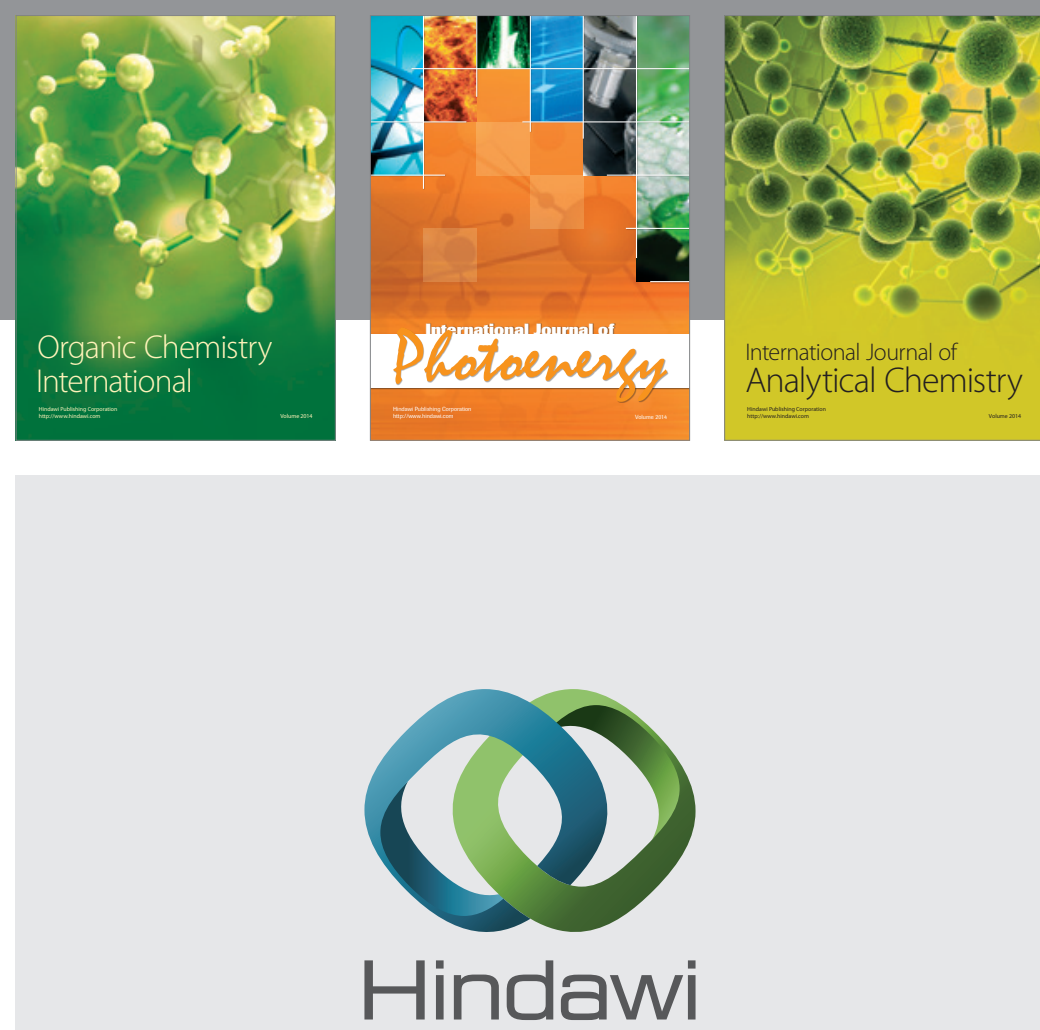

Submit your manuscripts at

http://www.hindawi.com
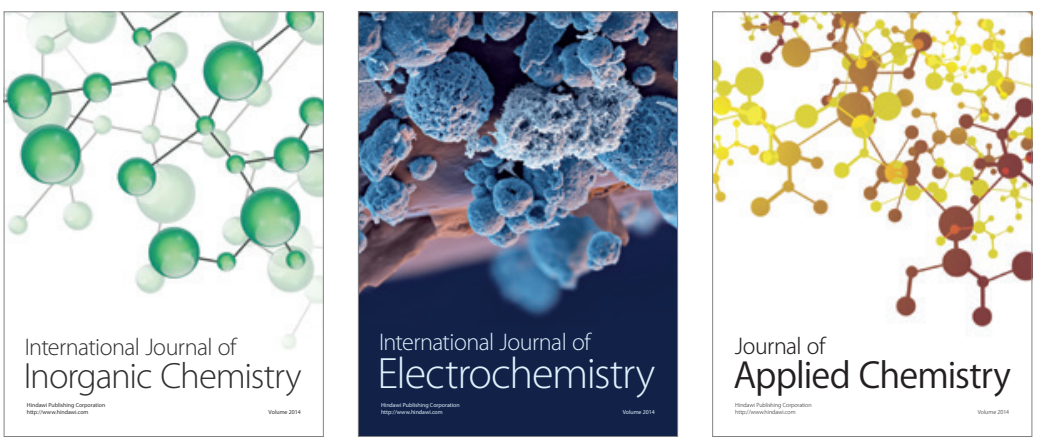

Journal of

Applied Chemistry
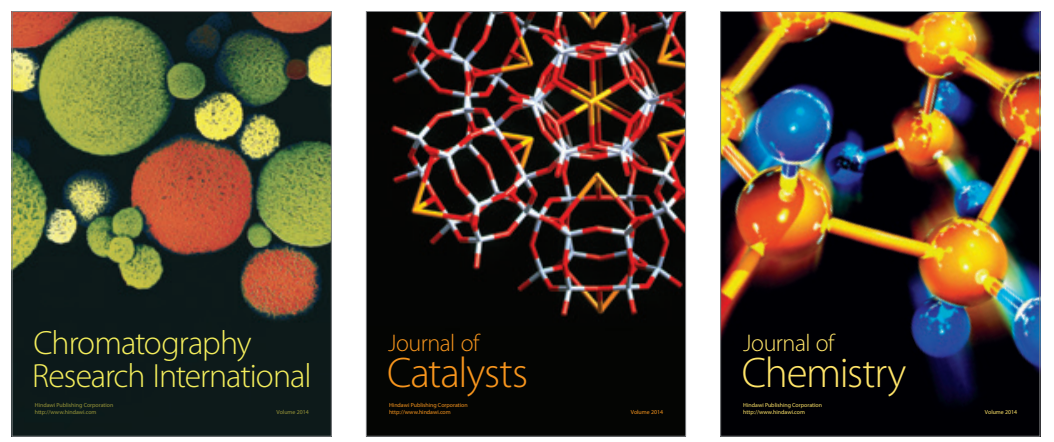
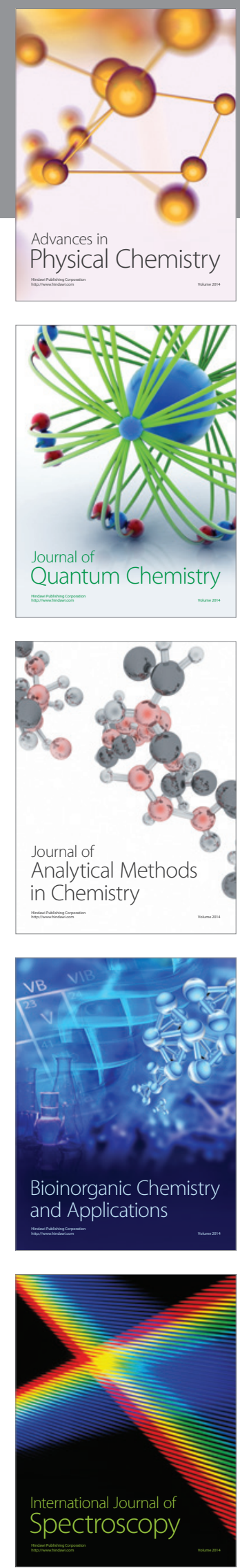